\title{
Adjuvant oestrogen treatment increases bone mineral density in postmenopausal women with rheumatoid arthritis
}

\author{
Hans R van den Brink, Willem F Lems, Amalia A van Everdingen, Johannes W J Bijlsma
}

\begin{abstract}
Objectives-The beneficial effect of oestrogens on bone mineral density in women with osteoporosis is well known. Patients with rheumatoid arthritis (RA) are at risk for osteoporosis. A study was therefore set up to investigate the effects of adjuvant oestrogen treatment on bone metabolism and bone mineral density in postmenopausal women with $R A$.

Methods-Forty postmenopausal women with active RA were admitted to a placebo controlled, double blind study investigating the beneficial effect of adjuvant oestradiols or placebo on bone metabolism and bone mineral density. Thirty three patients completed 52 weeks of treatment.

Results-At the start both treatment groups were comparable for all parameters. In the oestrogen group serum concentrations of osteocalcin decreased and concentrations of sex hormone binding globulin increased during the study. Bone mineral density measured by dual energy $x$ ray absorptiometry increased significantly in the lumbar vertebral spine and femoral neck in the oestrogen group compared with the placebo group.

Conclusions-This study shows that the use of adjuvant oestrogens in postmenopausal women with active RA increases bone mineral density.
\end{abstract}

(Ann Rheum Dis 1993; 52: 302-305)

Department of

Rheumatology,

University Hospital

Utrecht,

Utrecht, The

Netherlands

$H \mathrm{R}$ van den Brink

W F Lems

J W J Bijlsma

Deventer and Zutphen

Hospitals,

The Netherlands

A A van Everdingen

Correspondence to:

Dr H R van den Brink, Department of

Rheumatology F02.223,

University Hospital Utrecht,

PO Box 85500,

3508 GA Utrecht,

The Netherlands.

Accepted for publication

17 December 1992
Rheumatoid arthritis (RA) is often associated with considerable periarticular and generalised bone loss which may lead to an increased incidence of fractures. ${ }^{1-5}$ Several mechanisms are thought to contribute to bone loss in women with RA. Joint inflammation may lead to periarticular bone loss due to immobility and the release of local bone resorbing factors. ${ }^{46}$ Factors such as physical inactivity due to active RA, the use of glucocorticoids, and postmenopausal status may all contribute to generalised bone loss. ${ }^{1-4}$

After the menopause bone remodelling is increased with accelerated bone loss. Treatment with oestrogens can prevent this bone loss and in retrospective studies oestrogens reduce the incidence of vertebral ${ }^{7}$ and peripheral fractures $^{8}$ in healthy postmenopausal women. Epidemiological and immunological evidence suggests that female hormones may have a beneficial effect on the course of RA. ${ }^{9}$ To investigate the effects of oestrogens on bone metabolism and bone mineral density in postmenopausal women with RA, we conducted a randomised double blind placebo controlled study for one year.

\section{Patients and methods}

PATIENTS

After approval of the ethical committee 40 postmenopausal women with adult onset RA were admitted to the study; no contraindication for oestrogen treatment was found in any of the patients. Patients were considered to be postmenopausal when there had been no menstruation in the previous year. Patients using corticosteroids or hormone replacement treatment were not allowed to participate in the study. None of the patients used any drug known to influence bone metabolism. All patients fulfilled the revised criteria for $R^{10}$ and had active disease which met at least three of the following criteria: more than six painful joints; more than three swollen joints; erythrocyte sedimentation rate $>28 \mathrm{~mm} /$ hour; and morning stiffness for longer than 45 minutes. Disease modifying antirheumatic drugs were used in constant dosages for at least three months before the start of the study and were kept stable during the trial.

STUDY DESIGN

In a double blind controlled study we compared $2 \mathrm{mg}$ oestradiol valerate (Progynova) with placebo for 52 weeks. Oestradiol valerate and placebo were provided in identical capsules. The capsules were taken in cycles of 90 days. For the last 10 days of each cycle of 90 days all patients received $200 \mathrm{mg}$ progesterone (Progestan) daily to induce a withdrawal bleeding. After each cycle of 90 days all trial drugs were discontinued for one week. The complete study consisted of four cycles.

\section{EVALUATION}

Laboratory assessment of calcium metabolism and bone mineral density was determined at the start of the trial, after six months, and after 12 months. The following parameters were measured: calcium in serum and urine; serum 
alkaline phosphatase and albumin; creatinine in serum and urine; and hydroxyproline in urine. Compliance was measured by the concentration of serum sex hormone binding globulin. Sex hormone binding globulin increases significantly during oestrogen treatment.

Bone mineral density of the lumbar spine (L2-4) and femoral neck was determined by dual photon $x$ ray absorptiometry (Hologic QDR 1000, Waltham, MA, USA). The vertebral spine was scanned at the level of L2-4 and the hips were scanned at the femoral neck. The bone mineral density $\left(\mathrm{g} / \mathrm{cm}^{2}\right)$ was derived by dividing the bone mineral content of each region by the projected bone area. The precision, expressed as the coefficient of variation of duplicate measurements, was $1 \%$ for the lumbar spine and $1 \%$ for the femoral neck.

\section{STATISTICS}

Statistical analysis was carried out with the number cruncher statistical system (NCSS) software package version 5.1. Whenever appropriate two sample $t$ tests (within groups) or unpaired $t$ tests (between groups) were used for the comparison of data with a normal distribution. If skewness existed in the distribution of data the Wilcoxon rank sum test (within groups) or Mann-Whitney $U$ test (between groups) was used.

Table 1 Characteristics of the two treatment groups at start of the study. Values are mean (SD)

\begin{tabular}{llc}
\hline Characteristics & Oestrogen $(n=20)$ & Placebo $(n=20)$ \\
\hline Age (years) & $61(5)$ & $63(8)$ \\
Disease duration (years) & $9 \cdot 1(4 \cdot 2)$ & $9 \cdot 0(5 \cdot 7)$ \\
American Rheumatism Association functional class & $2 \cdot 2$ & $2 \cdot 1$ \\
Ritchie articular index & $16(5)$ & $17(6)$ \\
Erythrocyte sedimentation rate (mm/hour) & $37(15)$ & $33(15)$ \\
Weight (kg) & $65(12)$ & $66(10)$ \\
Disease modifying antirheumatic drugs & 1 & 2 \\
Antimalarial drugs & 9 & 8 \\
Sulphasalazine & 3 & 2 \\
Gold (by mouth) & 2 & 1 \\
Gold (intramuscular) & 2 & 4 \\
Methotrexate & 3 & 3 \\
None & & \\
\hline
\end{tabular}

\section{Results}

PATIENTS

Table 1 gives the characteristics of the two patient groups at the start of the trial. The two groups were comparable for all parameters. Seven of the 40 patients who entered the trial withdrew from the study before completing the full 52 weeks. Five of the patients were in the oestrogen group and two in the placebo group. Patients who dropped out from the study were included in the results until withdrawal. No clear effect of oestrogens on disease activity was seen.

\section{INDICES OF BONE METABOLISM}

Table 2 gives the results of parameters of bone metabolism before, after six, and after 12 months in serum and urine. There was a decrease in serum osteocalcin $(p<0.005)$ in the oestrogen group, indicating a decrease of osteoblastic activity. Parameters of bone resorption, urinary hydroxyproline, and calcium excretion decreased in the oestrogen group, but this was not statistically significant. Sex hormone binding globulin increased $(p<0.005)$ in the oestrogen group compared with the placebo group, indicating the use of oestrogens. None of the other parameters of calcium metabolism showed any change during the study in the two groups.

BONE MINERAL DENSITY MEASUREMENTS

Bone mineral density measurements of the lumbar spine and femoral neck in the two groups at the start, after six, and after 12 months are shown in table 2 . The bone mineral density of the lumbar spine and femoral neck increased in the oestrogen group whereas a decrease in the bone mineral density of the lumbar spine was seen in the placebo group. Percentage changes in the bone mineral density from baseline values in the two treatment groups were calculated and compared with each other; these results are shown in the figure. A significant difference between the two treatment groups occurred after six months in the bone mineral density of

Table 2 Parameters of bone metabolism and bone density $\left(\mathrm{g} / \mathrm{cm}^{2}\right)$ in the two treatment groups at the start of the study, after six, and after 12 months. Values are mean (SD)

\begin{tabular}{|c|c|c|c|c|c|c|}
\hline & \multicolumn{3}{|l|}{ Oestrogen } & \multicolumn{3}{|l|}{ Placebo } \\
\hline & Start & Six months & 12 months & Start & Six months & 12 months \\
\hline \multicolumn{7}{|l|}{ Serum } \\
\hline Calcium $(\mathrm{mmol} / \mathrm{l})$ & $2.42(0.07)$ & $2 \cdot 40(0 \cdot 10)$ & $2.43(0.11)$ & $2.38(0.08)$ & $2.39(0.06)$ & $2.37(0 \cdot 10)$ \\
\hline Creatinine $(\mu \mathrm{mol} / \mathrm{l})$ & $80(21)$ & $81(19)$ & $76(15)$ & $79(13)$ & $80(13)$ & $79(15)$ \\
\hline Alkaline phosphatase (U/I) & 75 (17) & $67(15)$ & $73(20)$ & $76(21)$ & 77 (23) & $79(23)$ \\
\hline Albumin $(g / 1)$ & $36(5)$ & $35(4)$ & $35(4)$ & $36(4)$ & $36(4)$ & $36(3)$ \\
\hline Osteocalcin $(\mu \mathrm{g} / 1)$ & $8 \cdot 1(2 \cdot 9)$ & $6 \cdot 8(1 \cdot 7) \dagger$ & $5 \cdot 8(1 \cdot 1) \ddagger$ & $8 \cdot 3(2 \cdot 4)$ & $8.9(2 \cdot 0)$ & $8 \cdot 3(2 \cdot 5)$ \\
\hline SHBG $(\mathrm{nmol} / /)^{\star}$ & $51(15)$ & $87(34) \leqq$ & $84(26) 5$ & $55(21)$ & $55(23)$ & $57(20)$ \\
\hline \multicolumn{7}{|l|}{ Urine } \\
\hline $\mathrm{Ca}$ /creatinine & $0.71(0.81)$ & $0.59(0.71)$ & $0.37(0.21)$ & $0.47(0.27)$ & $0.46(0.42)$ & $0.39(0 \cdot 26)$ \\
\hline $\begin{array}{l}\text { Hydroxyproline/creatinine } \\
\text { Byesysity }\end{array}$ & $22(11)$ & $19(6 \cdot 4)$ & $18(8 \cdot 7)$ & $21(7 \cdot 6)$ & $23(9 \cdot 7)$ & $25(8 \cdot 7)$ \\
\hline \multicolumn{7}{|l|}{ Bone density } \\
\hline $\begin{array}{l}\text { Lumbar spine } \\
\text { Femoral neck (R) }\end{array}$ & $\begin{array}{l}0.96(0.17) \\
0.63(0.08)\end{array}$ & $\begin{array}{l}0.99(0.16) \\
0.65(0.08) t\end{array}$ & $\begin{array}{l}1.00(0.17) d \\
0.65(0.08) t\end{array}$ & $\begin{array}{l}0.98(0.17) \\
0.69(0.10)\end{array}$ & $\begin{array}{l}0.97(0.16) \dagger \\
0.68(0.12)\end{array}$ & $\begin{array}{l}0.96(0.18) t \\
0.67(0.11)\end{array}$ \\
\hline Femoral neck (L) & $0.63(0.08)$ & $0.65(0.08) \dagger$ & $0.65(0.08) \dagger$ & $0.70(0 \cdot 11)$ & $0.69(0.11)$ & $0.69(0.11)$ \\
\hline
\end{tabular}

*SHBG=sex hormone binding globulin.

$\mathrm{tp}<0.05$.

$\neq \mathrm{p}<0.01$.

$\mathrm{Sp}<0.005$

$\mathrm{p}$ Value indicates indicates comparison from baseline value within the same treatment group. 
Percentage change in bone mineral density of $(A)$ the lumbar spine, $(B)$ the right, and $(C)$ the left femoral neck from baseline values in the two treatment groups. Differences in percentage change between the two treatment groups were calculated. ${ }^{\star} p<0.05$; ${ }_{*}^{*} p<0.01$; and $\star * * p<0.005$.

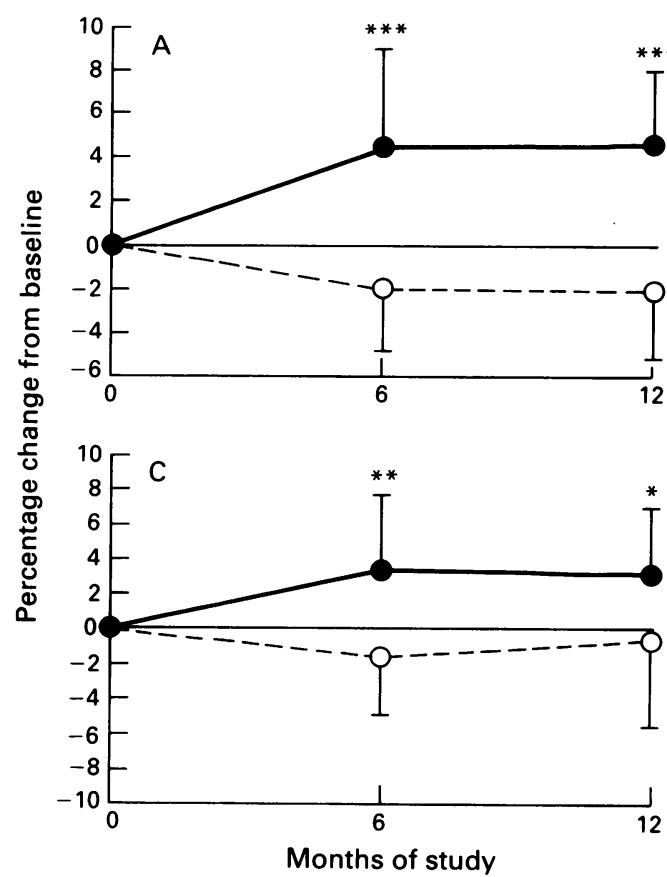

the lumbar spine $(p<0.005)$ and femoral neck (right $\mathrm{p}<0.05$ and left $\mathrm{p}<0.01$ ). In the oestrogen group a plateau was reached between six and 12 months after an initial increase in bone mineral density.

\section{Discussion}

Localised bone loss around the inflamed joint is a characteristic radiological sign of early RA. This (juxta-articular) osteoporosis is the result of stimulation of prostaglandin and cytokine synthesis by the inflammation which increases bone resorption. ${ }^{46}$ Generalised osteoporosis in patients with $\mathrm{RA}$ is believed to be multifactorial: impaired mobility, disease duration and activity, postmenopausal female status, and the use of glucocorticoids are regarded as the main factors. ${ }^{2-4}$ Other factors such as calcium malabsorption, vitamin D deficiency, reduced serum levels of dehydroepiandrosterone sulphate and increased systemic cytokines may also contribute to generalised osteoporosis. ${ }^{2} 1112$ Osteoporosis in patients with RA may lead to an increased risk of fractures. ${ }^{5}$ The patients we studied were at risk for osteoporosis for several reasons: their postmenopausal status, duration of RA, and disease activity.

In healthy women treatment with oestrogens can prevent osteopenia and reduce the incidence of spinal and peripheral fractures. ${ }^{78}$ The effects of adjuvant oestrogen treatment on parameters of bone metabolism and bone mineral density in patients with RA has not been reported before, however. This prospective study in postmenopausal women with RA shows that bone metabolism is positively influenced by oestrogens. The decrease in osteocalcin after six months in patients receiving oestrogens indicates a decrease in osteoblastic activity during treatment. Oestrogens are known to reduce bone resorption, leading to a decrease in

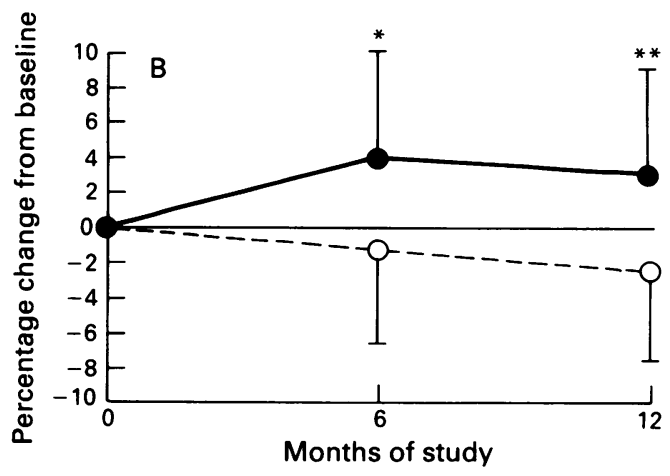

Oestrogens

Placebo urinary excretion of calcium and hydroxyproline, parameters of bone resorption. We did not find a significant change in these ratios in the oestrogen group, but the two ratios seemed to decrease.

Adjuvant oestrogen treatment had a beneficial effect on the bone mineral density of the lumbar spine and femoral neck. An increase of bone mineral density in the oestrogen group compared with the placebo group was seen after six months of treatment. An increase in bone mass was followed by a plateau in the lumbar spine and femoral neck, an effect also reported in other studies using drugs such as etidronate and calcitonin with an effect against bone resorption. ${ }^{13} 14$ An explanation for this phenomenon is that the resorption cavities are filled, increasing the bone mineral content, after a decrease in bone remodelling. After the empty resorption cavities are filled, no further increment in bone mass occurs and the plateau phase is observed. ${ }^{15}$ The bone mineral density in the lumbar spine increased more than in the femoral neck; this is because the lumbar spine consists of trabecular bone only, which is metabolically more active than cortical bone, the main substance of the femoral neck. An effect of oestrogens on bone metabolism is thus detected earlier in the lumbar spine than in the femoral neck.

The precise mechanisms by which oestrogens influence bone metabolism are still unclear but the net result is an increase in bone mineral density. This must be due to a change in the balance between bone formation and resorption, leading to a stronger inhibition of bone resorption than bone formation. Oestrogens may act directly on osteoblasts since receptors for oestrogens have been shown, ${ }^{16}$ but an indirect regulatory mechanism through growth factors has also been proposed. ${ }^{17}$ Recruitment of osteoblasts and osteoclasts from precursor cells is regulated by 
local and systemic growth factors. It has been suggested that replacement treatment with oestrogens leads to a decrease in plasma insulin-like growth factor I and an increase in plasma growth hormone.

In conclusion, this study shows that adjuvant treatment with oestrogens $(2 \mathrm{mg}$ oestradiol valerate) given for one year in postmenopausal women with RA influences bone metabolism and significantly increases the bone mineral density in the lumbar spine and femoral neck.

This work was supported by the Dutch League Against Rheumatism, 'National Reumafonds'. We thank all th participating patients and $\mathrm{Dr}$ Siewertsz van Reesema, help and advice, as well as Schering and Organon for providing the oestrogens and progesteron.

1 Sambrook P N, Eisman J A, Champion G D, et al. Determinants of axial bone loss in rheumatoid arthritis. Arthritis Rheum 1987; 30: 721 .

2 Bijlsma J W J. Bone metabolism in patients with rheumatoid arthritis. Clin Rheumatol 1988; 7: 16-23.

3 Dequeker J, Geusens P. Osteoporosis and arthritis. Ann Rheum Dis 1990; 49: 276-80.

4 Joffe I, Epstein S. Osteoporosis associated with rheumatoid arthritis: pathogenesis and management. Semin Arthritis Rheum 1991; 20: 256-72.

5 Hooyman J R, Melton L J, Nelson A M, O'Fallon W M Riggs B L. Fractures after rheumatoid arthritis. Arthritis Rheum 1984; 27: 1353-61.
6 McDonald B R, Gowen M. Cytokines and bone. $\mathrm{Br} \mathcal{F}$ Rheumatol 1992; 31: 149-55.

7 Ettinger B, Genant H K, Cann C E. Long-term estrogen replacement therapy prevents bone loss and fractures. Ann Intern Med 1985; 102: 319-24.

8 Kiel D P, Felson D T, Anderson J J, Wilson P W F Moskowitz M A. Hip fracture and the use of estrogens in postmenopausal women. The Framingham study. $N$ in postmenopausal women. The

9 van den Brink H R, Bijlsma J W J. Can oestrogens be used as adjuvant therapy in female patients with rheumatoid as adjuvant therapy in female patients with rheum

10 Arnett F C, Edworthy S M, Bloch D E, et al. The American Arnett F C, Edworthy S M, Bloch D E, et al. The American
Rheumatism Association 1987 revised criteria for the classification of rheumatoid arthritis. Arthritis Rheum 1988; 31: 315-24

11 van Soesbergen R M, Lips P, van den Ende A, van der Kors $\mathrm{J} \mathrm{K}$. Bone metabolism in rheumatoid arthritis compared with postmenopausal osteoporosis. Ann Rheum Dis 1986; 45: $149-55$.

12 Sambrook P N, Eisman J A, Champion G D, Pocock N A Sex hormone status and osteoporosis in postmenopausal women with rheumatoid arthritis. Arthritis Rheum 1988; 31: 973-8.

13 Gruber H E, Ivey J L, Baylink D J, et al. Long-term calcitonin therapy in postmenopausal osteoporosis. calcitonin therapy in post

14 Storm T, Thamsborg G, Steiniche T, Genant H K, Sörensen O H. Effect of intermittent cyclical etidronate therapy on bone mass and fracture rate in women with postmenopausal osteoporosis. N Engl f Med 1990; 322 1265-71.

15 Parfitt A M. Morphologic basis of bone mineral measurements: transient and steady state effects of treatment in osteoporosis. Miner Electrolyte Metab 1980; 4: $273-87$.

16 Eriksen E F, Colvard D S, Berg N J, et al. Evidence of estrogen receptors in normal osteoblast-like cells. Science 1988; 241: 84-6.

17 Duursma S A, Jaszmann L J, De Raadt M E, et al. Insulinlike growth factor- 1 and growth hormone during oestrogen replacement therapy. Growth Regulation. In press. 